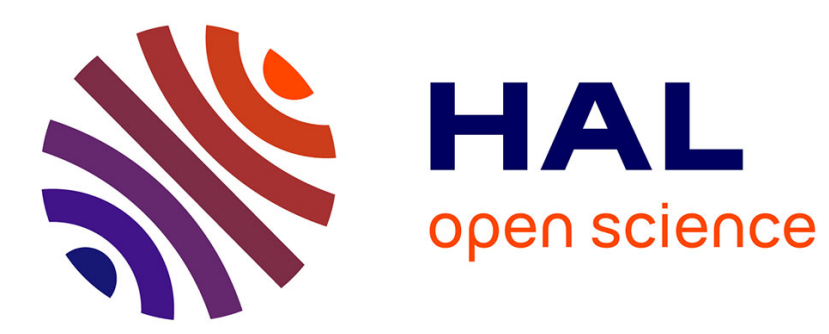

\title{
Reflection and transmission of transient ultrasonic wave in fractal porous material: Application of fractional calculus
}

\author{
Zine El Abiddine Fellah, M. Fellah, E. Ogam, A. Berbiche, C. Depollier
}

\section{- To cite this version:}

Zine El Abiddine Fellah, M. Fellah, E. Ogam, A. Berbiche, C. Depollier. Reflection and transmission of transient ultrasonic wave in fractal porous material: Application of fractional calculus. Wave Motion, 2021, 106, pp.102804. 10.1016/j.wavemoti.2021.102804 . hal-03310651

\section{HAL Id: hal-03310651 \\ https://hal.science/hal-03310651}

Submitted on 30 Jul 2021

HAL is a multi-disciplinary open access archive for the deposit and dissemination of scientific research documents, whether they are published or not. The documents may come from teaching and research institutions in France or abroad, or from public or private research centers.
L'archive ouverte pluridisciplinaire HAL, est destinée au dépôt et à la diffusion de documents scientifiques de niveau recherche, publiés ou non, émanant des établissements d'enseignement et de recherche français ou étrangers, des laboratoires publics ou privés. 


\section{Reflection and Transmission of Transient Ultrasonic Wave in Fractal Porous Material : Application of Fractional Calculus.}

Z.E.A. Fellah

LMA, CNRS, UPR 7051, Aix-Marseille Univ, Centrale Marseille, F-13402 Marseille Cedex 20, France

M. Fellah

Laboratoire de Physique Théorique, Faculté de Physique, USTHB, BP 32 El Alia, Bab Ezzouar 16111, Algérie.

E. Ogam

LMA, CNRS, UPR 7051, Aix-Marseille Univ, Centrale Marseille, F-13402 Marseille Cedex 20, France

A. Berbiche

Laboratoire de Physique Théorique, Faculté de Physique, USTHB, BP 32 El Alia, Bab Ezzouar 16111, Algérie.

C. Depollier

LUNAM Universite du Maine. UMR CNRS 6613 Laboratoire d'Acoustique de l'Universite du Maine UFR STS Avenue O. Messiaen 72085 Le Mans CEDEX 09 France. 


\section{ABSTRACT}

This paper provides a time domain model for the propagation of transient ultrasonic waves in a self-similar porous material having a rigid frame. This model is based on the formalism of Stillinger-Palmer-Stavrinou, which consists in modeling the fractal material as a porous medium with a non-integer dimensional space. This paper is devoted to the time-domain analytical calculus of the reflection and transmission operators that are expressed in terms of MittagLeffler functions. A sensitivity numerical study using ultrasonic reflected and transmitted waves is performed, highlighting the effect of of the material's physical parameters (fractal dimension, tortuosity, viscous characteristic length and porosity) on the waveforms.

PACS number : 43.20. Bi. 


\section{Introduction}

Natural media and many engineering materials have highly complicated structures [1-5]. Many porous materials possess a self-similar structure exhibiting scale invariance over many length scales [6-8]. For such materials, classical homogenization methods become inapplicable because heterogeneities play an important role on almost all scales [9-12].

The self-similar material can be defined as a medium with non-integer (fractal) mass dimension [1,13-15]. The non-integer Hausdorff dimension is the main characteristic of self similar media and materials but does not fully reflects of the specific properties of these media.

The self-similar structure of real materials cannot be observed on all scales, but only for discrete sizes of inhomogeneities belonging to an interval $\left[r_{\min }, r_{\max }\right]$ where $r_{\min }$ (resp $r_{\max }$ ) is the smallest (resp. the largest) scale [16] present in the object. Self-similarity is a typical property of fractal objects. Fractal $[17,18]$ sets are a particular case of self-similar sets.

The fractal porous material can be characterized by the relation between the mass $M\left(W_{s}\right)$ of a spherical region $W_{s}$ of this material and the radius $R$ of this region, in the form $[14,15]$

$$
M\left(W_{s}\right)=\mathcal{C} R^{D_{m}}
$$

where $\mathcal{C}$ is a constant. The parameter $D_{m}$ is called the mass dimension $[19,20]$ of the fractal medium, and is generally a non-integer less than 3. Anisotropic fractal materials can be characterized by the power-law relation for the mass of the parallelepiped region $W_{p}$ in the form $[14]$

$$
M\left(W_{p}\right)=\mathcal{C} L_{x}^{d_{1}} L_{y}^{d_{2}} L_{z}^{d_{3}}
$$

where the parameter $d_{k}$ is non-integer dimension along $X_{k}$-axis, $k=1,2,3$, and $L_{x}, L_{y}, L_{z}$ represent three edges that meet at one vertex. The parameter $d_{k}$ describes how to increase the medium mass in the case of increasing the size of the parallelepiped along one axis, when the parallelepiped sizes along other axes do not change. The sum $D_{m}=d_{1}+d_{2}+d_{3}$ is called the fractal mass dimension of the anisotropic fractal medium. 
An important conclusion about the behavior of the self similar material can be obtained using continuum models with a non-integer dimensional space [14,15,18,21-27]. Johnson et al [28] introduced the notion of fractal to describe the roughness of the surface pores (fluid-structure interface) and to calculate a correction of the viscous attenuation in a porous material due to this roughness. The propagation in porous media with fractal structure was also considered Wilson [29].

In 1973, Wilson [30] suggested the axioms for integrals in a non-integer dimensional space. Four years later, Stillinger [31] introduced a mathematical basis of integration in spaces with noninteger dimensions and generalized the Laplace operator in these spaces. Svozil [32] developed the product measure method, extended later by Palmer and Stavrinou [33] to multiple variables and different degrees of confinement in orthogonal directions.

In this work, we calculate, analytically and numerically, the reflection and transmission operators in the time domain for ultrasonic propagation in a self-similar porous material of non-integer dimension. The Stillinger-Svozil-Palmer-Starvinou formalism is used to derive the reflection and transmission coefficients first in the Laplace domain and then in the time domain by analytically performing the inverse Laplace transform. In our model, the pore space is a fractal and the matrix is non-fractal, so that we can then use the classical expressions for the dynamic tortuosity and compressibility to describe the visco-thermal losses in a fractal porous material. The sensitivity study allows us to highlight important conclusions on the effect of each physical parameter (porosity, tortuosity, viscous characteristic length, fractal dimension) on the reflected and transmitted waveforms.

\section{Model}

C. Palmer and P.N. Stavrinou [33] have generalized the formalism of Stillinger [31] for a noninteger dimensional space to $n$ orthogonal coordinates. The Euler Lagrange equations have been 
derived using the principle of least action in such spaces. The integral calculus in a non-integer dimensional space $[31,33]$ is greatly facilitated with the method of dimensional regularization $[31,33]$. The calculus of an integral over an area of non-integer dimension $D$ in the direction $x$, where : $(0 \leq D \leq 1)$, can be done with a regularization of the measure using the following relation $[32,34]$

$$
d^{D} x=\sigma_{D}(x) d x, \quad \text { where } \quad \sigma_{D}(x)=\frac{\pi^{\frac{D}{2}} x^{D-1}}{\Gamma(D / 2)}
$$

where $\Gamma$ is the Gamma function.

The principle of least action, based on the variational method, makes it possible to determine the equations of motion by doing an integral calculation. This method [35,37] is used to establish the equation of propagation of a wave in a porous medium along a direction $x$, of non-integer dimension $D$, the generalisation to a space consisting of three directions can be done immediately thanks to the product of measurements [34]. The corresponding Lagrangian $L$ is a functional of the field $\psi(x, t)$ considered (which can be a pressure field for example), and of its temporal and spatial derivatives which we note $\partial_{t} \psi(x, t)$ and $\partial_{x} \psi(x)$.

$$
\mathcal{S}=\int_{t_{i}}^{t_{f}} L\left(\psi(x, t), \partial_{t} \psi(x, t), \partial_{x} \psi(x, t)\right) d t
$$

Expressed in terms of the Lagrangian density $l$, the action is written as

$$
\mathcal{S}=\int_{t_{i}}^{t_{f}} d t \int_{\Omega} l\left(\psi(x, t), \partial_{t} \psi(x, t), \partial_{x} \psi(x, t)\right) d^{D} x
$$

where $d^{D} x$ represents the measurement in the $x$ direction and $\Omega$ the limits of the integration space. Since the dimension of the space in this direction is not integer, the measure is given by the relation (1).

The variational method consists in making an infinitesimal variation of the exact solution sought, as $\psi_{0}(x, t)$ assuming that its values at times $t_{i}$ and $t_{f}$ remain unchanged. An infinitely close solution is obtained which is given, together with its spatial and temporal derivatives, by the 
following expressions

$$
\begin{aligned}
& \psi(x, t)=\psi_{0}(x, t)+\delta \psi(x, t), \\
& \partial_{t} \psi(x, t)=\partial_{t} \psi_{0}(x, t)+\delta\left(\partial_{t} \psi(x, t)\right), \\
& \partial_{x} \psi(x, t)=\partial_{x} \psi_{0}(x, t)+\delta\left(\partial_{x} \psi(x, t)\right) .
\end{aligned}
$$

The principle of least action states that the action must remain stationary for small changes in the fields $\psi(x, t)$, which is expressed as

$$
\delta \mathcal{S}=\int_{t_{i}}^{t_{f}} d t \int_{\Omega} d x\left(\sigma_{D}(x) \frac{\partial \mathcal{L}}{\partial \psi} \delta \psi+\sigma_{D}(x) \frac{\partial \mathcal{L}}{\partial\left(\partial_{t} \psi\right)} \delta\left(\partial_{t} \psi\right)+\sigma_{D}(x) \frac{\partial \mathcal{L}}{\partial\left(\partial_{x} \psi\right)} \delta\left(\partial_{x} \psi\right)=0\right) .
$$

By reversing the differentiation and integrating by parts the last two terms of the above equation, we obtain

$\int_{t_{i}}^{t_{f}} d t \int_{\Omega} d x\left[\sigma_{D}(x) \frac{\partial \mathcal{L}}{\partial \psi}-\sigma_{D}(x) \partial_{t}\left(\frac{\partial \mathcal{L}}{\partial\left(\partial_{t} \psi\right)}\right)-\sigma_{D}(x) \partial_{x}\left(\frac{\partial \mathcal{L}}{\partial\left(\partial_{x} \psi\right)}\right)-\frac{\partial \mathcal{L}}{\partial\left(\partial_{x} \psi\right)} \partial_{x}\left(\sigma_{D}(x)\right)\right] \delta \psi=0$

knowing that this equation is valid whatever the variation of $\delta \psi$, and that

$$
\partial_{x}\left(\sigma_{D}(x)\right)=\left(D_{1}-1\right) \frac{\pi^{\frac{D_{1}}{2}} x^{D-2}}{\Gamma\left(\frac{D_{1}}{2}\right)}=\frac{D-1}{x} \sigma_{D}(x) .
$$

The Euler-Lagrange equation obtained for a medium of non-integer dimension $D$ in a direction $x$ is given by $[33,35]$

$$
\frac{\partial \mathcal{L}}{\partial \psi}-\frac{\partial}{\partial x}\left(\frac{\partial \mathcal{L}}{\partial\left(\frac{\partial \psi}{\partial t}\right)}\right)-\left(\frac{D-1}{x}\right) \frac{\partial \mathcal{L}}{\partial\left(\frac{\partial \psi}{\partial x}\right)}=0
$$

where $\mathcal{L}$ is the Lagrangian density. When $D=1$, the classical Euler Lagrange equation for a medium of integer dimension is found.

In the case of a homogeneous porous material with a rigid frame, which is a particular case of the Biot Theory [36], the expression of the Lagrangian density is given by [35,37]

$$
\mathcal{L}=\frac{1}{2}\left[\rho\left(\frac{\partial u}{\partial t}\right)^{2}-K_{a}\left(\frac{\partial u}{\partial x}\right)^{2}\right],
$$

where $u$ is the particle displacement, $\rho$ is the fluid density and $K_{a}$ is the adiabatic bulk modulus of the fluid. 
The equations of acoustics in the equivalent fluid model for a non-fractal porous material $(D=1)$ are given by [37]

$$
\rho \frac{\partial^{2} u}{\partial t^{2}}=-\frac{\partial p}{\partial x}, \quad p=-K_{a} \frac{\partial u}{\partial x}
$$

where $p$ is the acoustic pressure.

Taking into account the fluid-structure exchanges in the porous material, the density and compressibility of the fluid are "renormalized" by the tortuosity operator $\widetilde{\alpha}(t)$ and the compressibility operator $\widetilde{\beta}(t)$, via the relations $: \rho \rightarrow \rho \widetilde{\alpha}(t)$ and $K_{a} \rightarrow K_{a} / \widetilde{\beta}(t)$.

As was mentioned in Balankin etal [8], it is imperative to distinguish between three types of scale-invariant permeable media : 1) porous media in which the matrix is a fractal, whereas the pore space is non-fractal, (e.g. the Menger sponge and fractal aggregates) ; 2) fractally permeable media in which the pore space is a fractal, whereas the matrix is non-fractal, (e.g. the inverse Menger sponge and media with fractal pore and/or fracture networks);3) materials with the fractal matrix-pore interfaces, e.g. fracture surfaces. In this study, we are in the second case, in which the pore space is a fractal and the matrix is non-fractal, since $M(W)=\mathcal{C} R^{D_{M}}$ is the mass of fluid which is self-similar, we can thus use the expressions of the tortuosity and compressibility $\widetilde{\alpha}(t)$ and $\widetilde{\beta}(t)$ used for classical porous materials. Fig. 1 shows an example of a self-similar microstructure of a porous material.

In the asymptotic domain corresponding to the high frequency range, the expressions of $\widetilde{\alpha}(t)$ and $\widetilde{\beta}(t)$ are given by $[37,39,40]$

$\tilde{\alpha}(t)=\alpha_{\infty}\left(\delta(t)+\frac{2}{\Lambda}\left(\frac{\eta}{\pi \rho_{f}}\right)^{1 / 2} t^{-1 / 2}\right), \quad \tilde{\beta}(t)=\left(\delta(t)+\frac{2(\gamma-1)}{\Lambda^{\prime}}\left(\frac{\eta}{\pi \operatorname{Pr} \rho_{f}}\right)^{1 / 2} t^{-1 / 2}\right)$.

In these equations, $\operatorname{Pr}$ is the Prandtl number, $\delta(t)$ is the Dirac function, $\eta$ is the fluid viscosity, $\gamma$ is the adiabatic constant $\alpha_{\infty}$ is the tortusoity, $\Lambda$ and $\Lambda^{\prime}$ are the viscous and thermal characteristic lengths. In this model the time convolution of $t^{-1 / 2}$ with a function is interpreted as a semi derivative operator following the definition of the fractional derivative of order $\nu$ given by [41]

$$
D^{\nu}[x(t)]=\frac{1}{\Gamma(-\nu)} \int_{0}^{t}(t-u)^{-\nu-1} x(u) d u
$$


Using the Euler-Lagrange equation (Eq. 3), the expression of the Lagrangian (Eq. 4) and the viscous and thermal losses in the porous material (Eq. 5), we deduce the propagation equation for the porous material in a non-integer dimension space in the high frequency range [37]

$\frac{\partial^{2} p(x, t)}{\partial x^{2}}-\left(\frac{\rho \alpha_{\infty}}{K_{a}}\right) \frac{\partial^{2} p(x, t)}{\partial t^{2}}-\frac{2 \alpha_{\infty} \sqrt{\rho \eta}}{K_{a}}\left(\frac{2}{\Lambda}+\frac{2(\gamma-1)}{\Lambda^{\prime} \sqrt{P r}}\right) \frac{\partial^{3 / 2} p(x, t)}{\partial t^{3 / 2}}+\left(\frac{D-1}{x}\right) \frac{\partial p(x, t)}{\partial x}=0$

The first two terms, $\frac{\partial^{2} p(x, t)}{\partial x^{2}}$ and $\left(\frac{\rho \alpha \infty}{K_{a}}\right) \frac{\partial^{2} p(x, t)}{\partial t^{2}}$, describe the wave propagation with a wavefront speed $c=\sqrt{\frac{K_{a}}{\rho \alpha_{\infty}}}$, the third term, the fractional derivative on time, $\frac{2 \alpha_{\infty} \sqrt{\rho \eta}}{K_{a}}\left(\frac{2}{\Lambda}+\frac{2(\gamma-1)}{\Lambda^{\prime} \sqrt{P r}}\right) \frac{\partial^{3 / 2} p(x, t)}{\partial t^{3 / 2}}$ describes the attenuation of the wave due to the visco-thermal exchanges between fluid and structure, and finally the last term $\left(\frac{D-1}{x}\right) \frac{\partial p(x, t)}{\partial x}$ describes the self-similarity of the material via its fractal dimension $D$.

\section{Reflection and transmission problem}

The geometry of the problem is shown in Fig. 2. Consider a porous material of fractional dimension occupying the region $0 \leq x \leq L$. By considering that the incident sound wave is launched in the region $x \leq 0$, the expression of the acoustic field is given by

$$
p_{1}(x, t)=p^{i}(x, t)+p^{r}(x, t), \quad x<0,
$$

where the incident and reflected acoustic pressures at $x=0$ are respectively given by

$$
\begin{aligned}
& p^{i}(x, t)=\int_{0}^{t} p^{i}(\tau) \delta\left(t-\tau-\frac{x}{c_{0}}\right) d \tau, \\
& p^{r}(x, t)=\int_{0}^{t} \tilde{\mathcal{R}}(\tau) p^{i}\left(0, t-\tau+\frac{x}{c_{0}}\right) d \tau,
\end{aligned}
$$

where $: c_{0}=\sqrt{K_{a} / \rho}$ is the velocity in the free fluid, $\delta(t)$ is the Dirac function, $p^{i}(t)$ the incident signal and $\tilde{\mathcal{R}}$ the reflection operator in the time domain.

The second region (II) where : $0 \leq x \leq L$ is occupied by a porous medium of fractional spatial dimension, in which the acoustic wave obeys the propagation equation

$$
\frac{\partial^{2} p_{2}(x, t)}{\partial x^{2}}-\frac{1}{c^{2}} \frac{\partial^{2} p_{2}(x, t)}{\partial t^{2}}-e \int_{0}^{t} \frac{\partial^{2} p_{2}\left(x, t^{\prime}\right)}{\partial t^{\prime 2}} \frac{d t^{\prime}}{\sqrt{t-t^{\prime}}}+\left(\frac{D-1}{x}\right) \frac{\partial p_{2}(x, t)}{\partial x}=0,
$$


where $D$ is the fractional dimension $(0<D<1)$. The coefficients $c$ and $B$ are given by

$$
\frac{1}{c^{2}}=\left(\frac{\rho \alpha_{\infty}}{K_{a}}\right), \quad e=\frac{2 \alpha_{\infty} \sqrt{\rho \eta}}{K_{a}}\left(\frac{2}{\Lambda}+\frac{2(\gamma-1)}{\Lambda^{\prime} \sqrt{P r}}\right)
$$

In the third region $x \geq L$, the transmitted field is given by

$$
p_{3}(x, t)=\int_{0}^{t} \tilde{\mathcal{T}}(\tau) p\left(0, t-\tau-\frac{L}{c}-\frac{(x-L)}{c_{0}}\right) d \tau
$$

where $\tilde{\mathcal{T}}$ is the transmission operator in the time domain.

We will use the Laplace transform for the reflection and transmission problem in a slab of porous material of non-integer spatial dimension. Let us note by $P(x, z)$ the Laplace transform of $p(x, t)$, defined by

$$
P(x, z)=\mathcal{L}[p(x, t)]=\int_{0}^{\infty} \exp (-z t) p(x, t) d t
$$

The Laplace transform of the propagation equation (10) is given by

$$
\frac{d^{2} P_{2}(x, z)}{d x^{2}}+\left(\frac{D-1}{x}\right) \frac{d P_{2}(x, z)}{d x}-k^{2}(z) P_{2}(x, z)=0
$$

where

$$
k^{2}(z)=z^{2}\left(\frac{1}{c^{2}}+e \sqrt{\frac{\pi}{z}}\right)
$$

The Laplace transform of the field outside the slab is given by

$$
\begin{aligned}
& P_{1}(x, z)=\left[\exp \left(-z \frac{x}{c_{0}}\right)+R(z) \exp \left(z \frac{x}{c_{0}}\right)\right] P^{i}(z), \quad x \leq 0, \\
& P_{3}(x, z)=T(z) \exp \left[-\left(\frac{L}{c}+\frac{(x-L)}{c_{0}}\right) z\right] P^{i}(z), \quad x \geq L .
\end{aligned}
$$

Here $P_{1}(x, z)$ and $P_{3}(x, z)$ are respectively the Laplace transform of the field on the left and on the right of the slab, $P^{i}(z)$ denotes the Laplace transform of the incident field $p^{i}(t)$ and finally $\mathrm{R}(\mathrm{z})$ and $\mathrm{T}(\mathrm{z})$ are the Laplace transform of the reflection and the transmission operators, respectively.

Inside the material, the expression of the acoustic field in the Laplace domain is given by

$$
P_{2}(x, z)=a(z) x^{\nu} I_{\nu}[x k(z)]+b(z) x^{\nu} K_{\nu}[x k(z)],
$$


where $\nu=1-\frac{D}{2}$, as $0<D<1$; we deduce that $\frac{1}{2}<\nu<1$.

$I_{\nu}[x k(z)]$ and $K_{\nu}[x k(z)]$ are the modified Bessel functions. The coefficients $a(z)$ and $b(z)$ are determined using the continuity conditions of the acoustic field $P(x, z)$ at the interfaces $x=0$ and $x=L$ of the porous material

$$
P_{1}\left(0^{-}, z\right)=P_{2}\left(0^{+}, z\right) \quad \text { and } \quad P_{2}\left(L^{-}, z\right)=P_{3}(L+, z) .
$$

Using relations (15) and (16), we obtain :

$$
P_{1}\left(0^{-}, z\right)=(1+R(z)) P^{i}(z), \quad P_{3}\left(L^{-}, z\right)=T(z) \exp \left(-\frac{L}{c} z\right) P^{i}(z) .
$$

Taking into account the fact that $\lim _{x \rightarrow 0} x^{\nu} I_{\nu}(x k(z))=0$, and $\lim _{x \rightarrow 0} x^{\nu} K_{\nu}[x k]=\frac{2^{\nu-1} \Gamma(\nu)}{k^{\nu}}$ in Ref. 42, we deduce the expressions of $a(z)$ and $b(z)$

$$
b(z)=\frac{k^{\nu}(z)}{2^{\nu-1} \Gamma(\nu)} P_{1}\left(0^{-}, z\right), \quad a(z)=\frac{1}{L^{\nu} I_{\nu}(\operatorname{Lk}(z))} P_{3}\left(L^{-}, z\right)-b(z) \frac{K_{\nu}(L k(z))}{I_{\nu}(\operatorname{Lk}(z))} .
$$

The expression of the pressure field inside the material is then given by

$$
\begin{aligned}
P_{2}(x, z) & =\left(\frac{x}{L}\right)^{\nu} \frac{I_{\nu}(x k(z))}{I_{\nu}(L k(z))} P_{3}\left(L^{-}, z\right) \\
& +\frac{(x k(z))^{\nu}\left[I_{\nu}(L k(z)) K_{\nu}(x k(z))-I_{\nu}(x k(z)) K_{\nu}(L k(z))\right]}{2^{\nu-1} \Gamma(\nu) I_{n} u(L k(z))} P_{1}\left(0^{-}, z\right),
\end{aligned}
$$

where $P_{3}\left(L^{-}, z\right)$ and $P_{1}\left(0^{-}, z\right)$ are given by the relations $(18)$.

\section{Reflection and Transmission coefficients}

In this section, the reflection and transmission coefficients are derived in the Laplace domain, for a porous material of non-integer spatial dimension, using the boundary conditions of flow velocity at the interfaces $x=0$ and $x=L$.

\section{$2.1 \quad$ Interface $x=0$}

In the regions $(1)(x \leq 0)$ and $(2)(0 \leq x \leq L)$, the Euler equations are given by

$$
\begin{aligned}
& \left.\rho_{0} \frac{\partial v_{1}(x, t)}{\partial t}\right|_{x=0}=-\left.\frac{\partial p_{1}(x, t)}{\partial x}\right|_{x=0}, \\
& \left.\int_{0}^{t} \rho(t-\tau) \frac{\partial v_{2}(x, \tau)}{\partial \tau}\right|_{x=0} d \tau=-\left.\frac{\partial p_{2}(x, t)}{\partial x}\right|_{x=0},
\end{aligned}
$$


where $v_{1}(x, t)$ and $v_{2}(x, t)$ represent the acoustic velocity field in the regions (1) and (2), respectively. In Eq. $22 ; \rho(t)=\rho_{0} \widetilde{\alpha}(t)$ and $\widetilde{\alpha}(t)$ is given by Eq. 5 . The tortuosity operator is equal to 1 , in the free space (region (1)).

The flow continuity relation at $x=0$ is given by

$$
v_{1}(x, t)=\phi v_{2}(x, t)
$$

Using the Euler relations $(21,22)$ and the flow continuity relation (23), we obtain

$$
\left.\int_{0}^{t} \rho(t-\tau) \frac{\partial p_{1}(x, \tau)}{\partial x}\right|_{x=0} d \tau=\left.\phi \rho_{0} \frac{\partial p_{2}(x, t)}{\partial x}\right|_{x=0}
$$

In the Laplace domain, equation (24) is given by

$$
\rho(z)\left(\frac{z}{c_{0}}\right)(R(z)-1) P^{i}(z)=\left.\phi \rho_{0} \frac{\partial P_{2}(x, z)}{\partial x}\right|_{x=0}
$$

where $\rho(z)=\mathcal{L}[\rho(t)]$; is the Laplace transform of $\rho(t)$.

As the porous material is of non-integer dimension, the spatial derivative of the pressure field is given by

$$
\frac{\partial P_{2}(x, z)}{\partial x}=\frac{\partial P_{2}(x, z)}{\partial^{D} x} \frac{\partial^{D} x}{\partial x}
$$

the fractional derivative of order $D$ is obtained using the regularization method [34]

$$
\partial^{D} x=\frac{\pi^{\frac{D}{2}}|x|^{D-1}}{\Gamma\left(\frac{D}{2}\right)} \partial x=f(\nu) x^{1-2 \nu} d x, \quad \text { where } \quad f(\nu)=\frac{\pi^{1-\nu}}{\Gamma(1-\nu)} .
$$

Using (26) and (27), we obtain

$$
\frac{\partial P_{2}(x, z)}{\partial x}=f(\nu) x^{1-2 \nu} \frac{\partial^{D} P_{2}(x, z)}{\partial x}
$$

Knowing that

$$
\frac{\partial P_{2}(x, z)}{\partial_{D} x}=k(z) x^{\nu}\left[a(z) I_{\nu-1}(k x)-b(z) K_{\nu-1}(k x)\right]
$$

we obtain

$$
\frac{\partial P_{2}(x, z)}{\partial x}=k(z) f(\nu) x^{1-\nu}\left[a(z) I_{\nu-1}(k x)-b(z) K_{\nu-1}(k x)\right]
$$


By replacing $a(z)$ and $b(z)$ with their values (19)

$$
\begin{aligned}
& \frac{\partial P_{2}(x, z)}{\partial x}=k(z) f(\nu) x^{1-\nu} \times \\
& {\left[\frac{I_{\nu-1}(x k(z))}{L^{\nu} I_{\nu}(L k(z))} P_{3}\left(L^{-}, z\right)-\frac{k^{\nu}}{2^{\nu-1} \Gamma(\nu)} \frac{I_{\nu-1}(x k(z)) K_{\nu}(L k(z))+K_{\nu-1}(x k(z)) I_{\nu}(L k(z))}{I_{\nu}(L k(z))} P_{1}\left(0^{-}, z\right)\right] .}
\end{aligned}
$$

Using the following relations

$$
\begin{aligned}
& \lim _{x \rightarrow 0} x^{1-\nu} I_{\nu-1}(x k(z))=\frac{k^{\nu-1}(z)}{2^{\nu-1}} \frac{1}{\Gamma(\nu)} \\
K_{\nu-1}(x k(z))= & \frac{\pi}{2} \frac{I_{-\nu+1}(x k(z))-I_{\nu-1}(x k(z))}{\sin (\nu-1) \pi}=-\frac{\pi}{2} \frac{I_{-\nu+1}(x k(z))-I_{\nu-1}(x k(z))}{\sin \pi \nu} \\
= & -\frac{\Gamma(\nu) \Gamma(1-\nu)}{2}\left[I_{-\nu+1}(x k(z))-I_{\nu-1}(x k(z))\right]
\end{aligned}
$$

from where

$$
\lim _{x \rightarrow 0} x^{1-\nu} K_{\nu}(x k(z))=\frac{\pi(1-\nu)}{2^{\nu}} k^{\nu-1}
$$

one has

$$
\begin{aligned}
& \lim _{x \rightarrow 0} \frac{\partial P_{2}(x, z)}{\partial x}=\frac{k^{\nu}}{2^{\nu-1}} \frac{f(\nu)}{\Gamma(\nu)} \times \\
& {\left[\frac{1}{L^{\nu} I_{\nu}(L k(z))} P_{3}\left(L^{-}, z\right)-\left(\frac{k}{2}\right)^{\nu} \frac{K_{\nu}(L k(z))+\Gamma(\nu) \Gamma(1-\nu) I_{\nu}(L k(z))}{\Gamma(\nu) I_{\nu}(L k(z))} P_{1}\left(0^{-}, z\right)\right]}
\end{aligned}
$$

we also know that

$$
K_{\nu}(L k(z))+\Gamma(\nu) \Gamma(1-\nu) I_{\nu}(L k(z))=\Gamma(\nu) \Gamma(1-\nu) I_{-\nu}(L k(z)) .
$$

Therefore, we get

$$
\lim _{x \rightarrow 0} \frac{\partial P_{2}(x, z)}{\partial x}=\frac{k^{\nu}}{2^{\nu-1}} \frac{f(\nu)}{\Gamma(\nu)}\left[\frac{1}{L^{\nu} I_{\nu}(L k(z))} P_{3}\left(L^{-}, z\right)-\left(\frac{k}{2}\right)^{\nu} \Gamma(1-\nu) \frac{I_{\nu}(L k(z))}{I_{\nu}(L k(z))} P_{1}\left(0^{-}, z\right)\right] .
$$

Relation (25) is finally written

$$
-\frac{z \rho(z)}{c_{0}}(1-R(z))=\phi \rho_{0}\left(\frac{k}{2}\right)^{\nu} \frac{f(\nu)}{\Gamma(\nu)}\left[\frac{2}{L^{\nu} I_{\nu}(L k(z))} T(z) e^{-\frac{L}{c} z}-2\left(\frac{k}{2}\right)^{\nu} \Gamma(1-\nu) \frac{I_{\nu}(L k(z))}{I_{\nu}(L k(z))}(1+R(z))\right]
$$




\section{$2.2 \quad$ Interface $x=L$}

At the interface $x=L$, the Euler equation is written in the two regions (2) and (3) $(x \geq L)$ as

$$
\begin{aligned}
& \left.\int_{0}^{t} \rho(t-\tau) \frac{\partial v_{2}(x, \tau)}{\partial(\tau)}\right|_{x=L^{-}} d \tau=-\left.\frac{\partial p_{2}(x, t)}{\partial x}\right|_{x=L^{-}} \\
& \left.\rho_{f} \frac{\partial v_{3}(x, t)}{\partial t}\right|_{x=L^{+}}=-\left.\frac{\partial p_{3}(x, t)}{\partial x}\right|_{x=L^{+}} .
\end{aligned}
$$

The flow continuity relation at $x=L$ is given by

$$
v_{3}(x, t)=\phi v_{2}(x, t)
$$

Using the Euler relations $(33,34)$ and the flow continuity relation $(35)$, we obtain

$$
\left.\int_{0}^{t} \rho(t-\tau) \frac{\partial p_{3}(x, \tau)}{\partial x}\right|_{x=L} d \tau=\left.\phi \rho_{0} \frac{\partial p_{2}(x, t)}{\partial x}\right|_{x=L} .
$$

In the Laplace domain, equation (36) is given by

$$
-\frac{z}{c_{0}} \rho(z) P_{3}(L, z)=\left.\phi \rho_{0} \frac{\partial P_{2}(x, z)}{\partial x}\right|_{x=L} .
$$

Using the expression (28), relation (37) becomes

$$
\begin{aligned}
& -\frac{z \rho(z)}{c_{0}} T(z) \exp \left(-\frac{L}{c} z\right)=\phi \rho_{0} f(\nu) \\
& \times\left[\frac{k(z) I_{\nu-1}[L k(z)]}{L^{2 \nu-1} I_{\nu}[L k(z)]} P_{3}(L, z)-\left(\frac{k(z)}{2^{\nu-1}}\right)^{\nu} \frac{1}{\Gamma(\nu)} \frac{I_{\nu-1}(L k(z)) K_{\nu}(L k(z))+K_{\nu-1}(L k(z)) I_{\nu}(L k(z))}{L^{\nu-1} I_{\nu}(L k(z))}\right],
\end{aligned}
$$

and since

$$
I_{\nu-1}(L k(z)) K_{\nu}(L k(z))+K_{\nu-1}(L k(z)) I_{\nu}(L k(z))=\frac{1}{L k(z)}
$$

we obtain

$$
\frac{-z \rho(z)}{c_{0}} T(z) \exp \left(-\frac{L}{c} z\right)=\phi \rho_{0} f(\nu) \times\left[\frac{k(z) I_{\nu-1}(L k(z))}{L^{2 \nu-1} I_{\nu}(L k(z))} T(z) \exp \left(\frac{L}{c} z\right)-2\left(\frac{k(z)}{2 L}\right)^{\nu} \frac{(1+R(z))}{\Gamma(\nu) I_{\nu}(L k(z))}\right] .
$$

The equation system constituted by relations (32) and (38) gives the expressions of the reflection and transmission coefficients $R(z)$ and $T(z)$ in the Laplace domain

$$
\begin{aligned}
R(z) & =\frac{(\Psi(z)-\Phi(z))(\Phi(z)+\Omega(z))-\Theta(z) \Upsilon(z)}{\Theta(z) \Upsilon(z)-(\Phi(z)+\Psi(z))(\Phi(z)+\Omega(z))} \\
T(z) & =\frac{-2 \Theta(z) \Phi(z) \exp \left(-\frac{L}{c} z\right)}{\Theta(z) \Upsilon(z)-(\Phi(z)+\Psi(z))(\Phi(z)+\Omega(z))}
\end{aligned}
$$


where

$$
\begin{aligned}
& \Phi(z)=\frac{z \rho(z)}{c_{0}}, \quad \Upsilon(z)=\phi \rho_{0}\left(\frac{k(z)}{2}\right)^{\nu} \frac{f(\nu)}{\Gamma(\nu)} \frac{2}{L^{\nu} I_{\nu}(L k(z))} \\
& \Psi(z)=2\left(\frac{k(z)}{2}\right)^{\nu} \Gamma(1-\nu) \frac{I_{-\nu}(L k(z))}{I_{\nu}(L k(z))}, \quad \Omega(z)=\phi \rho_{0} f(\nu) \frac{k(z)}{L^{2 \nu-1}} \frac{I_{\nu-1}(L k(z))}{I_{\nu}(L k(z))} \\
& \Theta(z)=2\left(\frac{k(z)}{2 L}\right)^{\nu} \frac{1}{\Gamma(\nu) I_{\nu}(L k(z))}
\end{aligned}
$$

In the high frequency domain, corresponding to $z \rightarrow+\infty$, the system $(32,38)$ simplifies to

$$
\begin{aligned}
& \left(A+B z^{2 \nu-1}\right) R(z)-C z^{\nu-1 / 2} \exp (-L k(z)) \exp \left(-z \frac{L}{c}\right) T(z)=A-B z^{2 \nu-1} \\
& Q z^{\nu-1 / 2} \exp (-L k(z)) R(z)-H \exp \left(-\frac{L}{c} z\right) T(z)=-Q z^{\nu-1 / 2} \exp (-L k(z)),
\end{aligned}
$$

where the coefficients $A, B, C, Q$, and $H$ are given by

$$
\begin{gathered}
A=\frac{2^{\nu-1} c^{\nu} \Gamma(\nu) \alpha_{\infty}}{\phi c_{0} f(\nu)}, \quad B=\frac{\Gamma(1-\nu)}{(2 c)^{\nu}}, \quad C=\frac{\sqrt{2 \pi}}{L^{(\nu-1 / 2)} \sqrt{c}}, \\
Q=\frac{\sqrt{2 \pi}}{2^{(\nu-1)} c^{(\nu+1 / 2)} L^{(\nu-1 / 2)} \Gamma(\nu)}, \quad H=\frac{1}{c L^{(2 \nu-1)}}+\frac{\alpha_{\infty}}{\phi c_{0} f(\nu)} .
\end{gathered}
$$

The solution of the system (41) gives the expressions of the reflection $R(z)$ and transmission $T(z)$ coefficients in the high frequency domain

$$
\begin{aligned}
R(z) & =\frac{H\left(A-B z^{2 \nu-1}\right)+C Q z^{2 \nu-1} \exp (-2 L k(z))}{H\left(A+B z^{2 \nu-1}\right)-C Q z^{2 \nu-1} \exp (-2 L k(z))}, \\
T(z) & =\frac{2 A Q z^{\nu-1 / 2} \exp (-L k(z)) \exp \left(\frac{L}{c}\right)}{H\left(A+B z^{2 \nu-1}\right)-C Q z^{2 \nu-1} \exp (-2 L k(z))} .
\end{aligned}
$$

These expressions can be developed in series of powers of $\exp (-2 L k(z))$ to bring out the effect of the interfaces of the material

$$
\begin{aligned}
& R(z)=\frac{A-B z^{2 \nu-1}}{A+B z^{2 \nu-1}}+\frac{2 A}{A+B z^{2 \nu-1}} \sum_{n \geq 0}\left(\frac{C Q z^{2 \nu-1}}{H\left(A+B z^{2 \nu-1}\right)}\right)^{n+1} \exp (-2(n+1) L k(z)), \\
& T(z)=\frac{2 A Q z^{\nu-1 / 2} \exp \left(\frac{L}{c} z\right)}{H\left(A+B z^{2 \nu-1}\right)} \cdot \sum_{n \geq 0}\left(\frac{C Q z^{2 \nu-1}}{H\left(A+B z^{2 \nu-1}\right)}\right)^{n} \exp (-(2 n+1) L k(z))
\end{aligned}
$$

Relations (44) represent the general expressions of the reflection and transmission coefficients $R(z)$ and $T(z)$ taking into account the multiple reflections at the interfaces of the material $(x=0$ 
and $x=L)$.

While it is of theoretical interest to consider multiple reflections in transmission and reflection, in the experimental reality [40,43-46], a temporal windowing process is performed, which allows sensing the first transmitted wave and the reflected wave at the first interface of the porous material. This is to avoid interference from other signals that spuriously reflected by stray objects. These waves arrive shifted with a time delay. The second transmitted wave makes a return trip, in the material layer, as compared to the first transmitted wave, it is more attenuated (since air-saturated porous materials are quite absorbent). The reason for avoiding capturing it is because it interferes with the spurious undesirable reflections of the first transmitted wave with the receiving transducer and the material interface. It is therefore not always, particularly for resistive materials, direct to distinguish and separate them. The second transmitted wave's amplitude is very small and is embedded in the noise, making the signal-to-noise ratio very low. As for the reflected wave at the second interface within the porous layer, it makes one round trip more than the wave reflected by the first interface (the first wave instantly reflected by the material). The second wave reflected at the rear interface of the porous layer interferes with the wave reflected at the first interface and the other reflected by the receiver transducer. This wave is generally very attenuated after a round trip from its propagation in the resistive porous layer. We can therefore conclude that only the first transmitted wave and the wave reflected by the first interface of the porous material can be reliably exploitable experimentally. It is therefore not necessary to calculate the contributions of multiple reflections in transmission and reflection since we capture only the first wave.

Let us consider the simple case, where we only take into account the first transmitted and reflected waves by the interfaces of the porous material. It is then sufficient to retain the first 
term of the series appearing in the second members of $R(z)$ and $T(z)$

$$
\begin{aligned}
R(z) & =\frac{A-B z^{2 \nu-1}}{A+B z^{2 \nu-1}}+\frac{2 A C Q z^{2 \nu-1}}{H\left(A+B z^{2 \nu-1}\right)^{2}} \exp (-2 L k(z)), \\
T(z) & =\frac{2 A Q z^{\nu-1 / 2} \exp \left(\frac{L}{c} z\right)}{H\left(A+B z^{2 \nu-1}\right)} \exp (-L k(z)) .
\end{aligned}
$$

By replacing $z$ by $j \omega$, we find the expressions of the reflection and transmission coefficients in the frequency domain.

\section{Reflection and Transmission operators in the time domain}

To obtain the analytical expressions of the reflection and transmission operators in the time domain, it is necessary to calculate the inverse Laplace transforms of the reflection and transmission coefficients. The expression (45) can rewrite as

$$
R(z)=\frac{A}{B} \frac{1}{z^{2 \nu-1}+A / B}-\frac{z^{2 \nu-1}}{z^{2 \nu-1}+A / B}+\frac{2 A C Q}{B^{2} H} \frac{z^{2 \nu-1}}{\left(z^{2 \nu-1}+A / B\right)^{2}} \exp (-2 L k(z)) .
$$

The inverse Laplace transforms of the different terms of the reflection and transmission coefficients (Eqs. 46 and 47) are expressed using the Mittag-Leffler function [47] with two parameters (Appendix. A)

$$
E_{\vartheta, \zeta}(z)=\sum_{n \geq 0} \frac{z^{n}}{\Gamma(\vartheta n+\zeta)},
$$

where $\Gamma(x)$ is the Gamma function of the second kind. In addition, the inverse Laplace transform of $\exp (-l k(z))$ is given by : $\mathcal{L}^{-1}(\exp (-l k(z)))=G(t, l)$, which represents the Green function of the porous material (Appendix B).

We obtain the temporal expressions of the reflection and transmission operators

$$
\begin{aligned}
\tilde{\mathcal{R}}(t) & =\frac{A}{B} t^{2 \nu-2} E_{2 \nu-1,2 \nu-1}\left(-\frac{A}{B} t^{2 \nu-1}\right)-E^{1} E_{2 \nu-1,0}\left(-\frac{A}{B} t^{2 \nu-1}\right) \\
& +\frac{2 A C Q}{B^{2} E} \int_{0}^{t} \tau^{2 \nu-2} E_{2 \nu-1,0}^{1}\left(-\frac{A}{B} \tau^{2 \nu-1}\right) G\left(t-\tau, \frac{2 L}{c}\right) d \tau . \\
\tilde{\mathcal{T}}(t) & =\frac{2 A Q}{B E} \int_{0}^{t} \tau^{\nu-\frac{3}{2}} E_{2 \nu-1, \nu-\frac{1}{2}}\left(-\frac{A}{B} \tau^{2 \nu-1}\right) G\left(t-\tau+\frac{L}{c}, \frac{L}{c}\right) d \tau,
\end{aligned}
$$


where

$$
E_{\vartheta, \zeta}^{(1)}(z)=\frac{d}{d z} E_{\vartheta, \zeta}(z)
$$

The first two terms of the reflection operator : $\frac{A}{B} t^{2 \nu-2} E_{2 \nu-1,2 \nu-1}\left(-\frac{A}{B} t^{2 \nu-1}\right)-E^{1} E_{2 \nu-1,0}\left(-\frac{A}{B} t^{2 \nu-1}\right)$ depending on the Mittag-Leffler function represent the contribution of the first interface $x=0$, the third term : $\frac{2 A C Q}{B^{2} E} \int_{0}^{t} \tau^{2 \nu-2} E_{2 \nu-1,0}^{1}\left(-\frac{A}{B} \tau^{2 \nu-1}\right) G\left(t-\tau, \frac{2 L}{c}\right) d \tau$ corresponds to the contribution of the second interface $x=L$, this end wave reflected by the second interface of the slab makes a round trip inside the material and travels the distance 2L designated by the Green's function $G\left(t-\tau, \frac{2 L}{c}\right)$.

The transmission operator (50) designates the first direct transmitted wave having propagated in a material of thickness $\mathrm{L}$, and the Green function $G\left(t-\tau+\frac{L}{c}, \frac{L}{c}\right)$ describes this propagation.

\subsection{Integer dimensional space}

Let us consider the special case of the porous material of integer space dimension; $D=1$, which is equivalent to $\nu=\frac{1}{2}$, since : $\nu=1-\frac{D}{2}$, and by taking into account only the first reflection interface for the reflection coefficient and the first transmitted wave (Eqs. 45 and 46), we obtain the following relations in the Laplace domain

$$
R=\frac{A_{\left(\nu=\frac{1}{2}\right)}-B_{\left(\nu=\frac{1}{2}\right)}}{A_{\left(\nu=\frac{1}{2}\right)}+B_{\left(\nu=\frac{1}{2}\right)}}, \quad T=\frac{2 A_{\left(\nu=\frac{1}{2}\right)} Q_{\left(\nu=\frac{1}{2}\right)} \exp \left(\frac{L}{c} z\right)}{H_{\left(\nu=\frac{1}{2}\right)}\left(A_{\left(\nu=\frac{1}{2}\right)}+B_{\left(\nu=\frac{1}{2}\right)}\right)} \exp (-L k(z)) .
$$

For $\nu=\frac{1}{2}$, the coefficients $A, B, C, D$ and $H$ have the values :

$A_{\left(\nu=\frac{1}{2}\right)}=\sqrt{\frac{\pi c}{2}}\left(\frac{\alpha_{\infty}}{\phi c_{0}}\right), \quad B_{\left(\nu=\frac{1}{2}\right)}=\sqrt{\frac{\pi}{2 c}}, \quad C_{\left(\nu=\frac{1}{2}\right)}=\sqrt{\frac{2 \pi}{c}}, \quad Q_{\left(\nu=\frac{1}{2}\right)}=\frac{2}{c}, \quad H_{\left(\nu=\frac{1}{2}\right)}=\frac{1}{c}+\frac{\alpha_{\infty}}{\phi c_{0}}$.

which gives :

$$
R=\frac{\sqrt{\alpha_{\infty}}-\phi}{\sqrt{\alpha_{\infty}+\phi}}, \quad T=\frac{4 \sqrt{\alpha_{\infty}} \phi}{\left(\phi+\sqrt{\alpha_{\infty}}\right)^{2}} \exp (-L k(z))
$$

In the time domain, the expressions of the reflection and transmission operators are given by

$$
\tilde{\mathcal{R}}(t)=\frac{\sqrt{\alpha_{\infty}}-\phi}{\sqrt{\alpha_{\infty}+\phi}} \delta(t), \quad \tilde{\mathcal{T}}(t)=\frac{4 \sqrt{\alpha_{\infty}} \phi}{\left(\phi+\sqrt{\alpha_{\infty}}\right)^{2}} G\left(t+\frac{L}{c}, \frac{L}{c}\right)
$$


These expressions are exactly the same as those found in the literature for the reflection and transmission coefficients in a classical porous medium of integer dimension [40]. From the general expressions of the reflection and transmission coefficients (Eqs. 45 and 46) in a porous medium of non-integer spatial dimension, we find the particular case of classic porous mediums studied a few years ago [40].

\section{Numerical simulation and discussion}

In this section, numerical simulations of waves transmitted and reflected by a porous material of non-integer dimension are given and discussed. The above parameters are not, in general, independent, meaning that when one of them is varied the others will also varied in an unknown manner, but theses parameters are still varied in this work to be able to study their separarated influence on the transmitted and/or reflected waves. The physical properties of the material are : thickness $L=1 \mathrm{~cm}$, fractal dimension $D=0.45$, porosity $\phi=0.95$, tortuosity $\alpha_{\infty}=1.07$, viscous characteristic length $\Lambda=300 \mu m$, thermal characteristic length $\Lambda^{\prime}=900 \mu m$. The incident signal (Eq. 8) used in the simulations is given in Fig. 3.

Figure 4 shows the wave transmitted by a porous material of non-integer dimension for different values of the fractional dimension $D$. These transmitted signals are simulated by convolving the transmission operator (Eq. 50) with the incident signal (Eq. 8) given in Fig. 3, using relation (12). From Figure 4, we can see that the amplitude of the transmitted wave increases with the value of the non-integer dimension. However, this tendency is reversed for the large values of $\mathrm{D}$. Indeed, the transmitted wave corresponding to $D=0.95$ is more attenuated than the wave corresponding to $D=0.7$. We can thus say that there are two regimes : one valid for the low values of $D$ where the material is not very resistive, and another regime for the high values of $D$, where the porous material is resistive (more absorbent).

Figure 5 gives the numerical simulations of the transmitted waves for different values of the 
tortuosity $\alpha_{\infty}$. A significant change is observed on the amplitude and arrival time of the signals. The acoustic wave is all the more attenuated as the tortuosity is high. An important change is observed in the wave speed (time lag between the simulated signals). By increasing the value of the tortuosity $\alpha_{\infty}$, the signal arrives late, because its wavefront velocity $c$ decreases (Eq. 11). This phenomenon is also observed in the case of non-fractal porous media (of dimension $\mathrm{D}=1$ ) [40].

The sensitivity to porosity is studied in Figure 6. A significant change in signal amplitude is observed while the signal arrival time remains virtually unchanged. By decreasing the porosity, the proportion of the fluid in which the acoustic wave propagates in the porous medium decreases, making the medium more resistive and absorbent, which leads to a decrease in the amplitude of the transmitted wave. The velocity of the wavefront does not change because its expression (Eq. 11) does not depend on the porosity. The simulations in Figure 6 are done with a value of the fractal dimension $D=0.45$, by decreasing this value to $D=0.1$, the transmitted wave becomes completely insensitive to the porosity. We can therefore conclude that the sensitivity to porosity of the transmitted wave is all the more important as the value of the fractal dimension is large.

The sensitivity to viscous characteristic length $(\Lambda)$ in transmission is studied in Figure 7 . We observe that the transmitted wave is very sensitive to this parameter, which in fact describes the viscous acoustic interactions and losses in the material, and gives us an idea on the radius of the narrow pores associated with viscous exchanges. The amplitude of transmitted wave decreases as the viscous length decreases, which is the characteristic of absorbent materials. Note that the signals are more dispersive as the value of $\Lambda$ decreases. This is explained by the fact that this parameter intervenes in the coefficient $e$ (Eq. 11) of the fractional term of the propagation equation (Eqs. 7 and 10). The fractional derivative describes the temporal dispersion of the signal due to visco-thermal exchanges between the fluid and the structure of the fractal porous medium.

From this sensitivity study, we can conclude that fractal dimension, tortuosity, porosity and 
viscous characteristic length are very important parameters for ultrasonic wave propagation in fractal porous media. These parameters act differently on the transmitted waveform (amplitude, dispersion and wavefront velocity).

Let us now study the sensitivity of the reflected wave to the physical parameters, and more particularly the sensitivity of the wave reflected by the first interface, given by the first two terms of expression (49) of the reflection operator in the time domain, or the first term of the expression (45) of the reflection coefficient in the Laplace domain.

The numerical simulation of the reflected wave is obtained by convolving the reflection operator with the incident wave given in figure 3. The results (Fig. 8) show that by increasing the value of the non-integer dimension $D$, the wave reflected at the first interface decreases in amplitude (i.e., is more attenuated), which means that the material becomes less resistive. This tendency is observed in transmission except for large values of $D$ where the tendency is reversed in transmission.

The study of the sensitivity of the reflected wave to tortuosity, for a fixed value of the fractal dimension at $D=0.45$, reveals no variation of the wave for different values of tortuosity, however, for the value $D=0.99$ (close to non-fractal porous), the reflected wave becomes very sensitive to tortuosity (Fig. 9). By increasing the value of tortuosity, the medium becomes more resistive and therefore the amplitude of the reflected wave is greater. For this large value of the fractal dimension $(D=0.99)$, we get closer to the results of the non-fractal case $[45,46]$, however for lower values of $D$, the effect of the tortuosity becomes negligible.

Figures 10 and 11 show the effect of porosity on the reflected wave at the first interface. Figure 10 corresponds to the value of the fractal dimension $\mathrm{D}$ equal to 0.45 and figure 11 to $\mathrm{D}=0.99$. We note that in figure 10 the effect of porosity is not as important as in figure 11 . This result means that the effect of porosity is important in reflection for large values of the fractal dimension. This trend is similar to what we observed with tortuosity.

The wave reflected at the first interface is theoretically independent of the viscous charac- 
teristic length (Eqs. 45 and 49), and so the sensitivity study with respect to this parameter is unnecessary.

This sensitivity study using numerical simulations of transmitted and reflected waves has allowed to highlight the most influential physical parameters. In transmission, the effect of tortuosity and viscous characteristic length is important for both small and large values of the fractal dimension. However, the effect of porosity is not significant for small values of the fractal dimension. In reflection, the viscous characteristic length does not appear naturally in the expression of the reflected wave at the first interface. The sensitivity of the tortuosity and the porosity on the first reflected wave is significant for large values of the fractal dimension. Finally, the fractal dimension appears to be the most important parameter in both modes (reflected and transmitted) simultaneously, which leads us to conclude that this parameter is essential in the description of the acoustic propagation in fractal porous media and that it is necessary to develop methods to characterize it.

\section{CONCLUSION}

In this paper, ultrasound propagation in a fractal porous material with a rigid structure has been studied. The Stillinger-Palmer-Stavrinou formalism was used, in which the fractal porous material was modeled as a non-integer dimension material. The responses of the material were first derived in the Laplace domain by computing the reflection and transmission coefficients. Then, in a second step, analytical calculations of the inverse Laplace transform gave the reflection and transmission operators in the time domain, using the properties of Mittag-Leffler functions. Numerical simulations of the reflected and transmitted waves revealed the sensitivity of physical parameters describing the propagation such as fractal dimension, tortuosity, viscous characteristic length and porosity. The fractal dimension was the most influential parameter in both reflection and transmission. This work opens perspectives for solving direct and inverse 
problems in natural and artificial porous media with self-similarity. 


\section{APPENDIX. A}

The following inverse Laplace transforms depend on the Mittag-Leffler function $E_{\vartheta, \zeta}(z)$ (Eq. 48) :

$$
\begin{aligned}
& \mathcal{L}^{-1}\left(\frac{1}{z^{2 \nu-1}+\frac{A}{B}}\right)=t^{2 \nu-2} E_{2 \nu-1,2 \nu-1}\left(-\frac{A}{B} t^{2 \nu-1}\right) \\
& \mathcal{L}^{-1}\left(\frac{z^{2 \nu-1}}{z^{2 \nu-1}+\frac{A}{B}}\right)=t^{-1} E_{2 \nu-1,0}\left(-\frac{A}{B} t^{2 \nu-1}\right) \\
& \mathcal{L}^{-1}\left(\frac{z^{2 \nu-1}}{\left(z^{2 \nu-1}+\frac{A}{B}\right)^{2}}\right)=t^{2 \nu-2} E_{2 \nu-1,0}^{(1)}\left(-\frac{A}{B} t^{2 \nu-1}\right), \\
& \mathcal{L}^{-1}\left(\frac{z^{\nu-\frac{1}{2}}}{\left(z^{2 \nu-1}+\frac{A}{B}\right)}\right)=t^{\nu-\frac{3}{2}} E_{2 \nu-1, \nu-\frac{1}{2}}\left(-\frac{A}{B} t^{2 \nu-1}\right),
\end{aligned}
$$

where, relation (51) is used. 


\section{APPENDIX. B}

$\mathcal{L}^{-1}(\exp (-l k(z)))=G(t, l)$ is the inverse Laplace transform of $\exp (-l k(z))$, where 1 is a positive constant (thickness), given the Green function of the porous medium :

$$
G(t, l)=\left\{\begin{array}{l}
0 \quad \text { if } \quad 0 \leq t \leq \frac{l}{c}, \\
\Xi(t)+\Delta \int_{0}^{t-l / c} h(t, \xi) d \xi \quad \text { if } \quad t \geq \varphi,
\end{array}\right.
$$

with

$$
\Xi(t)=\frac{e^{\prime}}{4 \sqrt{\pi}} \frac{\varphi}{\left(t-\frac{l}{c}\right)^{3 / 2}} \exp \left(-\frac{e^{\prime 2} \varphi^{2}}{16\left(t-\frac{l}{c}\right)}\right),
$$

where $h(\tau, \xi)$ has the following form :

$$
h(\xi, \tau)=-\frac{1}{4 \pi^{3 / 2}} \frac{1}{\sqrt{(\tau-\xi)^{2}-l^{2} / c^{2}}} \frac{1}{\xi^{3 / 2}} \int_{-1}^{1} \exp \left(-\frac{\chi(\mu, \tau, \xi)}{2}\right)(\chi(\mu, \tau, \xi)-1) \frac{\mu d \mu}{\sqrt{1-\mu^{2}}}
$$

and where $\chi(\mu, \tau, \xi)=\left(\Delta \mu \sqrt{(\tau-\xi)^{2}-l^{2} / c^{2}}+e^{\prime}(\tau-\xi)\right)^{2} / 8 \xi, e^{\prime}=e c_{0}^{2} \sqrt{\pi}$, and $\Delta=\pi e^{2} c_{0}^{4}$, where : $e$ is given by Eq. 11 . 


\section{REFERENCES}

[1] J. Feder, Fractals, (Plenum Press, New York, 1988).

[2] K. Oleschko, G. Korvin, A.S. Balankin, R.V. Khachaturov, L. Flores, B. Figueroa, J. Urrutia, F. Brambila, Fractal scattering of microwaves from soils, Phys. Rev. Lett. 89, 18-28 (2002) 188501, https ://doi.org/10.1103/PhysRevLett.89.188501.

[3] A.S. Balankin,A. Hotra-Rangel, G. Garcia-Perez, F. Gayosso-Martinez, H. Sanchez-Chavez, C.L. Martinez Gonzalez, Fractal features of a crumpling network in randomly folded thin matter and mechanics of sheet crushing, Phys. rev. E 87 (2013) 052806, https://doi.org/10.1103/PhysRevE.87.052806.

[4] K. Liu, M. Ostadhassan, Quantification of the microstructures of Bakken shale reservoirs using multi-fractal and lacunarity analysis, J. Nat, Gas Sci. Eng. 39 62-71 (2017). https ://doi.org/10.1016/j.jngse.2017.01.035

[5] A.S. Balankin, Mapping physical problems on fractals onto boundary value problems within continuum framework, Physics Letter A, 382 141-146 (2018). https ://doi.org/10.1016/j.physleta.2017.11.005.

[6] G. Korvin, Fractal Models in the Earth Sciences, Elsevier, New York, 1992.

[7] M. Sahimi, Flow and Transport in porous media and Fractured rocks, 2nd ed., VHC Publications, Weinheim, 2011.

[8] A. S. Balankin, J-C. Valdivia, J. Marquez, O. Susarrey, M.A. Solorio-Avila, Anomalous diffusion of fluid momentum and Darcy-like law for laminar flow in media with fractal porosity, 
Physics Letters A 380 2767-2773 (2016)

[9) A. S. Balankin, A continuum framework for mechanics of fractal materials I : from fractional space to coninuum with fractal metric, Eur. Phys. J. B 8890 (2015). https ://doi.org/10.1140/epjb/e2015$60189-\mathrm{y}$.

[10] B. Yu, Analysis of flow in fractal porous media, Appl. Mech. rev. 61 365-372 (2008) 050801.

[11] A.S. Balankin, B.E. Elizarraraz, Map of fluid flow in fractal porous medium into fractal continuum flow, Phys. rev. E 85 (2012) https ://doi.org/10.1103/PhysRevE.85.056314

[12] M.N. Najafi, M. Ghaedi, Geometrical clusters of Darcy's reservoir model and ising universality class, Physica A 427 82-91 (2015)

[13] K.F. Falconer, The Geometry of Fractal Sets, (Cambridge University Press, 1985).

[14]V.E. Tarasov, Anisotropic fractal media by vector calculus in non-integer dimensional space, Journal of Mathematical Physics, 55, 083510 (2014); https ://doi.org/10.1063/1.4892155.

[15]V.E. Tarasov, Vector calculus in non-integer dimensional space and its applications to fractal media, Communications in Nonlinear Science and Numerical Simulation, 20, 360-374 (2015). https ://doi.org/10.1016/j.cnsns.2014.05.025.

[16] J. W. Dollinger, R. Metzler and T. F. Nonnenmacher, Bi-asymptotic fractals : fractals between lower and upper bounds, J. Phys. A. Math. Gen. 31, 3839-3847 (1998). 
[17] B. B. Mandelbrot, The Fractal Geometry of Nature, (updated and augmented ed., 468 pp., W. H. Freeman, New York, 1983).

[18] M.F. Barnsley, Fractals everywhere. (Morgan Kaufmann, San Mateo, 1993).

[19] F. Hausdorff, Dimension und äusseres Mass, Math. Ann. 79 , 157-179 (1919).

[20] D. Schleicher, Hausdorff dimension, its properties and its surprises , Am. Math. Monthly, 114, 509-528 (2007).

[21] P. N. Demmie, M. Ostoja-Starzewski, Waves in Fractals media, J. Elast. 104, 187-204 (2011).

[22] J. Li and M. Ostoja-Starzewski, Fractal solids, product measures and fractional wave equations, Proc. R. Soc. A 465, 2521-2536 (2009).

[23] M. Ostoja-Starzewski, Continuum mechanics models of fractal porous media : Integral relations and extremum principles, Journal of Mechanics of materials and structures. 4, 901-912 (2009).

[24] M. Ostoja-Starzewski, J. Li, H. Joumaa and P. N. Demmie, From fractal media to continuum mechanics, ZAMM, Z. Angew. Math. Mech. 94, 373-401 (2014).

[25] H. Joumaa and M. Ostoja-Starzewski, On the wave propagation in isotropic fractal media, Z. Angew. Math. Phys. 62, 1117-1129 (2011). 
[26] V.E. Tarasov, Wave equation for fractal solid string, Modern Physics Letters 19, 721-728 (2005).

[27] V.E. Tarasov, Acoustic waves in fractal media : non-integer dimensional spaces approach, Wave Motion 63, 18-22 (2016).

[28] D.L. Johnson, J. Koplik and R. Dashen, Theory of dynamic permeability and tortuosity in fluid-saturated porous media, J. Fluid. Mech. 176, 379-402 (1987).

[29] K. Wilson, Relaxation-matched modeling of propagation through porous media, including fractal pore structure, J. Acoust. Soc. Am. 94, 1136-1145 (1993).

[30] K.G. Wilson, Quantum field - theory models in less than 4 dimensions, Physical Review D 7 (10) 2911-2926 (1977).

[31] F.H. Stillinger, Axiomatic basis for spaces with non-integer dimensions, Journal of mathematical Physics 18(6) 2911-2926 (1973).

[32] K Svozil, Quantum field theory on fractal spacetime : a new regularisation method, J. Phys. A : Math. Gen. 20, 3861-3875 (1987).

[33] C. Palmer, P.N. Stavrinou, Equations of motion in a non-integer dimensional space, Journal of Physics A 37 (27) 698767003 (2004).

[34] S.I. Muslih and D. Baleanu, Mandelbrot scaling and parametrization invariant theories, Rom. Rep. Phys. 62 (4) 689-696 (2010). 
[35] A. Berbiche, M. Fellah, Z.E.A. Fellah, E. Ogam, F.G. Mitri, C. Depollier, Transient acoustic wave in self-similar porous material having rigid frame : Low frequency domain, Wave Motion, 68,12-21 (2016).

[36] M.A. Biot, Theory of Propagation of Elastic Waves in a Fluid-Saturated Porous Solid. II. Higher Frequency Range, J. Acoust. Soc. Am. 28, 179-191 (1956).

[37] M. Fellah, Z.E.A. Fellah, A. Berbiche, E. Ogam, F.G. Mitri and C. Depollier, Transient Ultrasonic Wave Propagation in Porous Material of non-integer space dimension, Wave Motion, 72,276-286 (2017).

[38] J. F. Allard, Propagation of Sound in Porous Media : Modeling Sound Absorbing Materials (Chapman and Hall, London, 1993).

[39] Z.E.A. Fellah and C. Depollier, Transient acoustic wave propagation in rigid porous media : A time-domain approach, J. Acoust. Soc. Am. 107, 683-688 (2000).

[40] Z.E.A. Fellah, M. Fellah, W. Lauriks, Direct and inverse scattering of transient acoustic waves by a slab of rigid porous material, J. Acoust. Soc. Am. 113, 61-72 (2013).

[41] S.G. Samko, A.A. Kilbas and O.I. Marichev, Fractional Integrals and Derivatives : Theory and Applications, ( Gordon and Breach Science Publishers, Amsterdam, 1993).

[42] G.B. Arfken and H.J. Weber, Mathematical methods for physicists, (Elsevier Academic Press, Inc, San Diego, 2005). 
[43] R. Roncen, Z.E.A. Fellah, E. Piot, F. Simon, E. Ogam, M. Fellah and C. Depollier, Inverse identification of a higher order viscous parameter of rigid porous media in the high frequency domain, J. Acoust. Soc. Am. 145, 1629-1639 (2019).

[44] R. Roncen, Z.E.A. Fellah, F. Simon, E. Piot, M. Fellah, E. Ogam, and C. Depollier, Bayesian inference for the ultrasonic characterization of rigid porous materials using reflected waves by the first interface, J. Acoust. Soc. Am. 144, 210-221 (2018).

[45] Z.E.A. Fellah, S. Berger, W. Lauriks, C. Depollier, C. Aristegui and J.Y. Chapelon, Measuring the porosity and the tortuosity of porous materials via reflected waves at oblique incidence, J. Acoust. Soc. Am. 113 (5), 2424-2433, (2003).

[46] Z.E.A. Fellah, S. Berger, W. Lauriks, C. Depollier, M. Fellah, Measuring the porosity of porous materials having a rigid frame via reflected waves : A time domain analysis with fractional derivatives, J. Appl. Phys., Vol. 93, (1), 296-303, (2003).

[47] K. Diethelm, N.J. Ford, A.D. Freed and Yu. Luchko, Algorithms for the fractional calculus : a selection of numerical methods, Comput. Methods. Appl. Mech. Eng. 194 743-773 (2005). 


\section{FIGURE CAPTIONS}

Figure 1. Example of a self-similar microstructure of a porous material, each pore splits into two pores when passing from one scale to another.

Figure 2. The geometry of the problem for unidirectional propagation in a porous slab occupying the space $0 \leq x \leq L$.

Figure 3. Incident signal used for numerical simulations.

Figure 4. (Color online) Variation of the ultrasonic waveform transmitted by a self-similar porous material for different values of the fractal dimension $D$, the values of the other parameters have been kept constant $\left(L=1 \mathrm{~cm}, \phi=0.95, \alpha_{\infty}=1.07, \Lambda=300 \mu m\right.$ and $\left.\Lambda^{\prime}=900 \mu m\right)$.

Figure 5. (Color online) Variation of ultrasonic waveform transmitted by a self-similar porous material for different values of the tortuosity $\alpha_{\infty}$, the values of the other parameters have been kept constant $\left(L=1 \mathrm{~cm}, \phi=0.95, D=0.45, \Lambda=300 \mu \mathrm{m}\right.$ and $\left.\Lambda^{\prime}=900 \mu \mathrm{m}\right)$.

Figure 6. (Color online) Variation of ultrasonic waveform transmitted by a self-similar porous material for different values of the porosity $\phi$, the values of the other parameters have been kept constant $\left(L=1 \mathrm{~cm}, \alpha_{\infty}=1.07, D=0.45, \Lambda=300 \mu \mathrm{m}\right.$ and $\left.\Lambda^{\prime}=900 \mu \mathrm{m}\right)$.

Figure 7. (Color online) Variation of ultrasonic waveform transmitted by a self-similar porous material for different values of the viscous characteristic length $\Lambda$, the values of the other parameters have been kept constant $\left(L=1 \mathrm{~cm}, \phi=0.95, \alpha_{\infty}=1.07, D=0.45\right.$ and $\left.\Lambda^{\prime}=900 \mu \mathrm{m}\right)$. 
Figure 8. (Color online) Variation of ultrasonic waveform reflected by a self-similar porous material for different values of the fractal dimension $D$, the values of the other parameters have been kept constant $\left(\phi=0.95, \alpha_{\infty}=1.07\right)$.

Figure 9. (Color online) Variation of ultrasonic waveform reflected by a self-similar porous material for different values of the tortuosity $\alpha_{\infty}$ and for a value of the fractal dimension : $D=0.99$, the value of porosity is $\phi=0.95$.

Figure 10. (Color online) Variation of ultrasonic waveform reflected by a self-similar porous material for different values of the porosity $\phi$ and for a value of the fractal dimension : $D=0.45$, the value of tortuosity is $\alpha_{\infty}=1.07$.

Figure 11. (Color online) Variation of ultrasonic waveform reflected by a self-similar porous material for different values of the porosity $\phi$ and for a value of the fractal dimension : $D=0.99$, the value of tortuosity is $\alpha_{\infty}=1.07$. 
FIG. 1

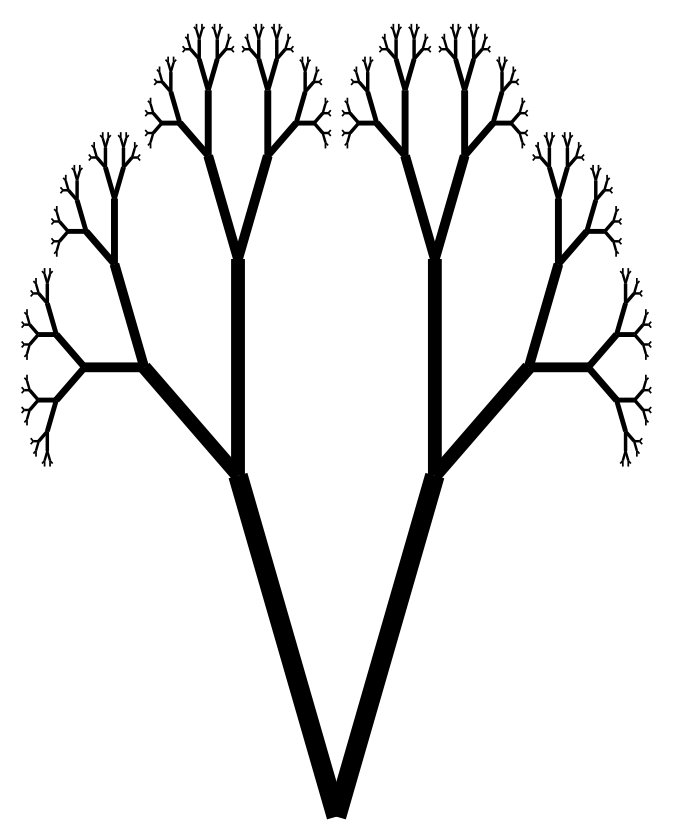


FIG. 2

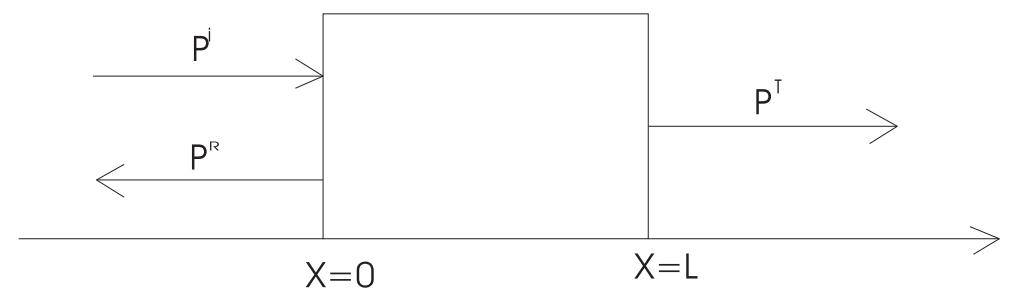


Figure 3

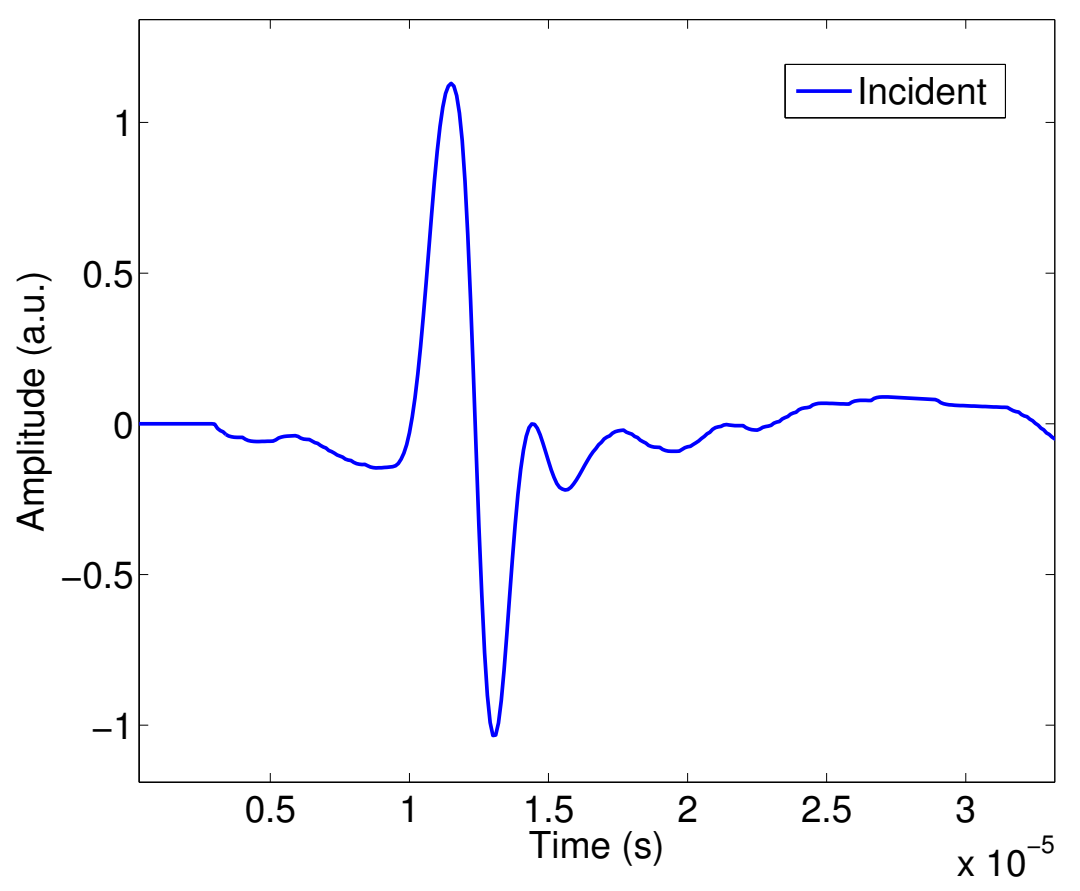


Figure 4

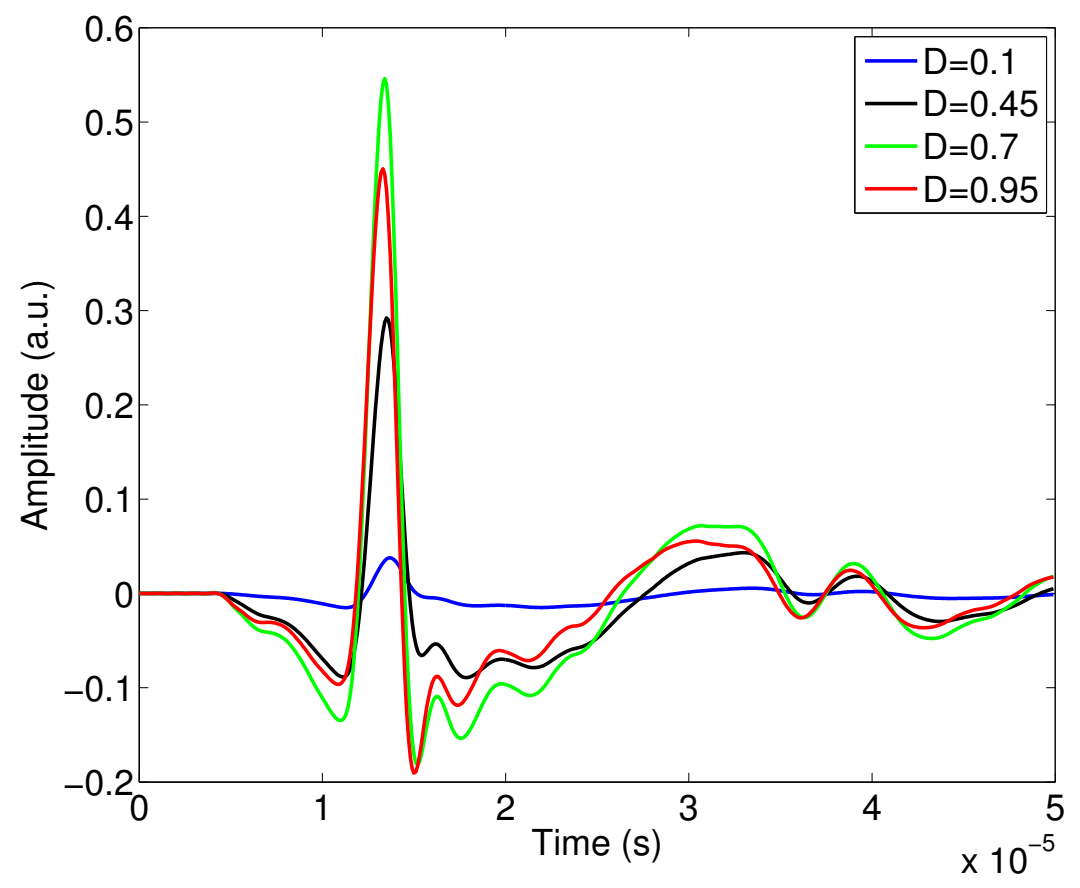


Figure 5

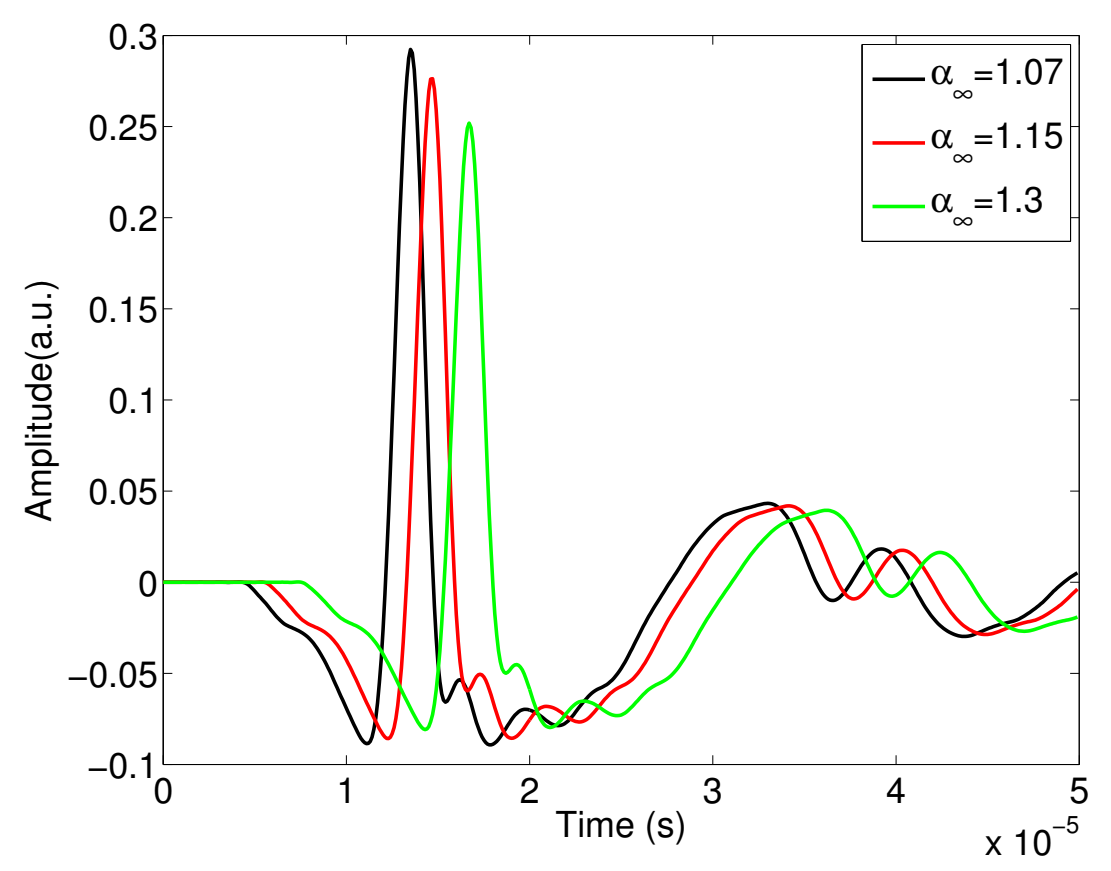


Figure 6

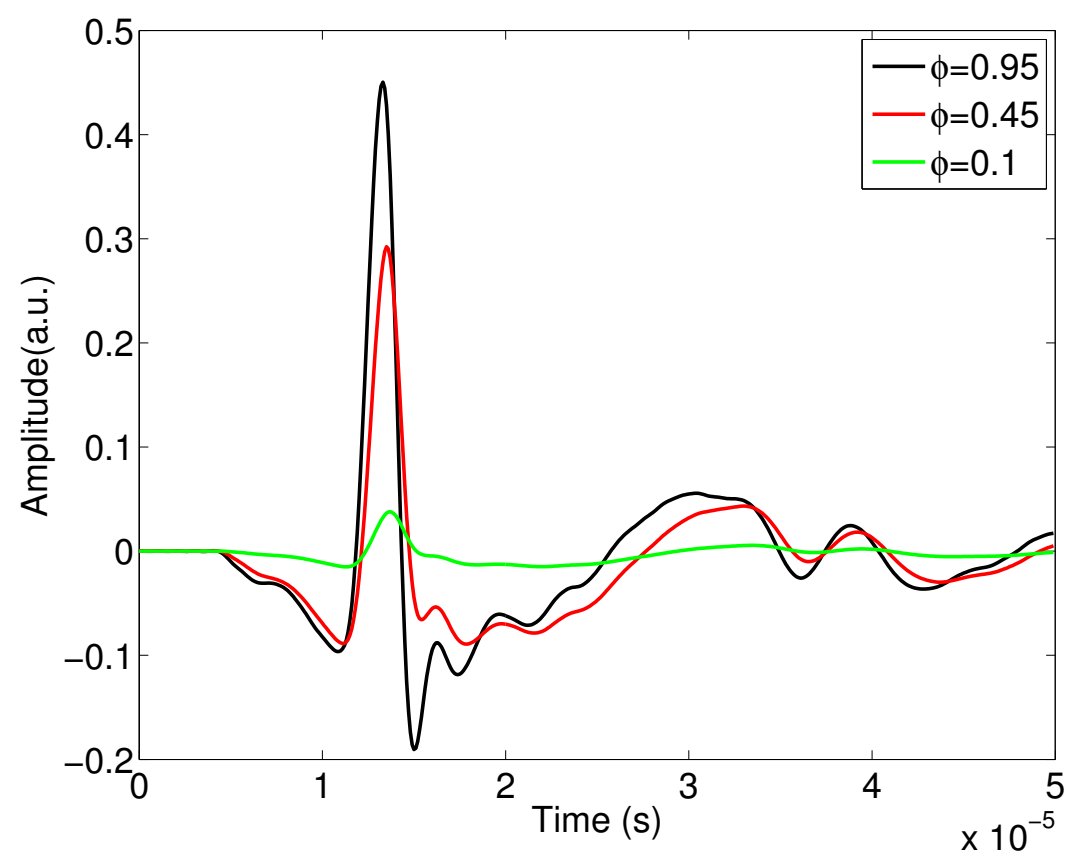


Figure 7

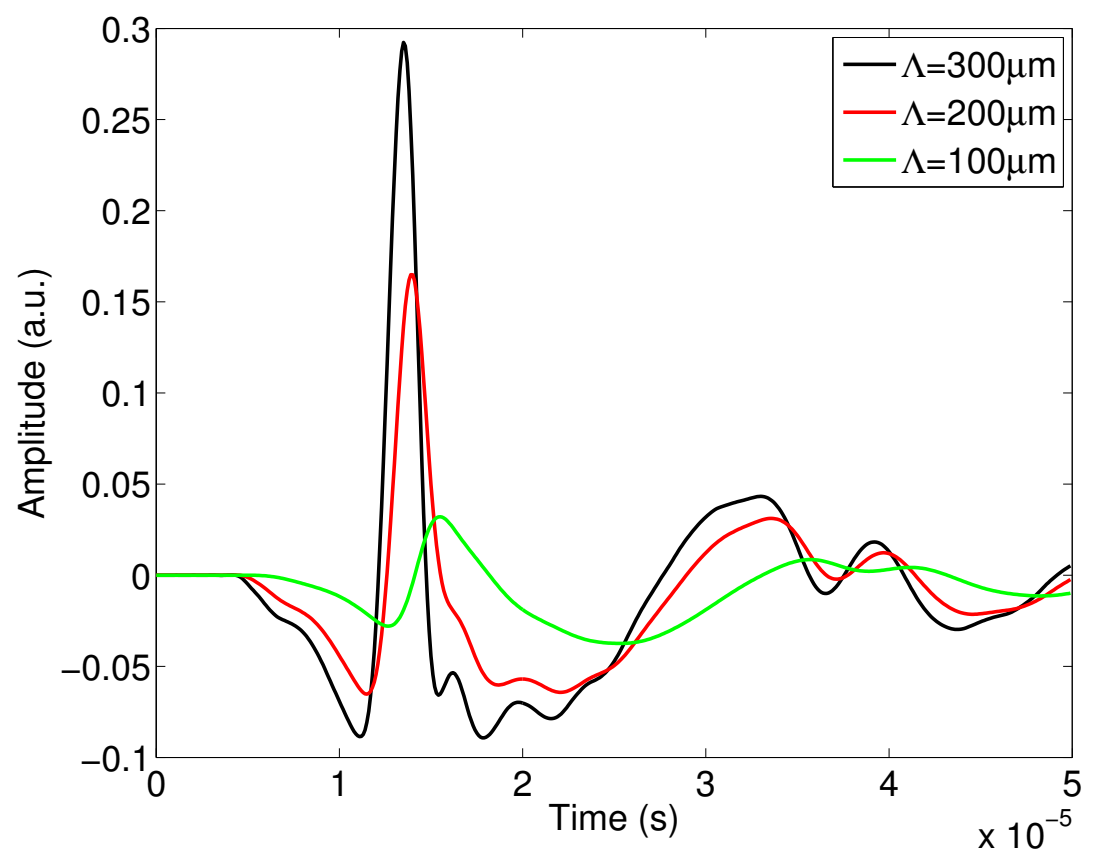


Figure 8

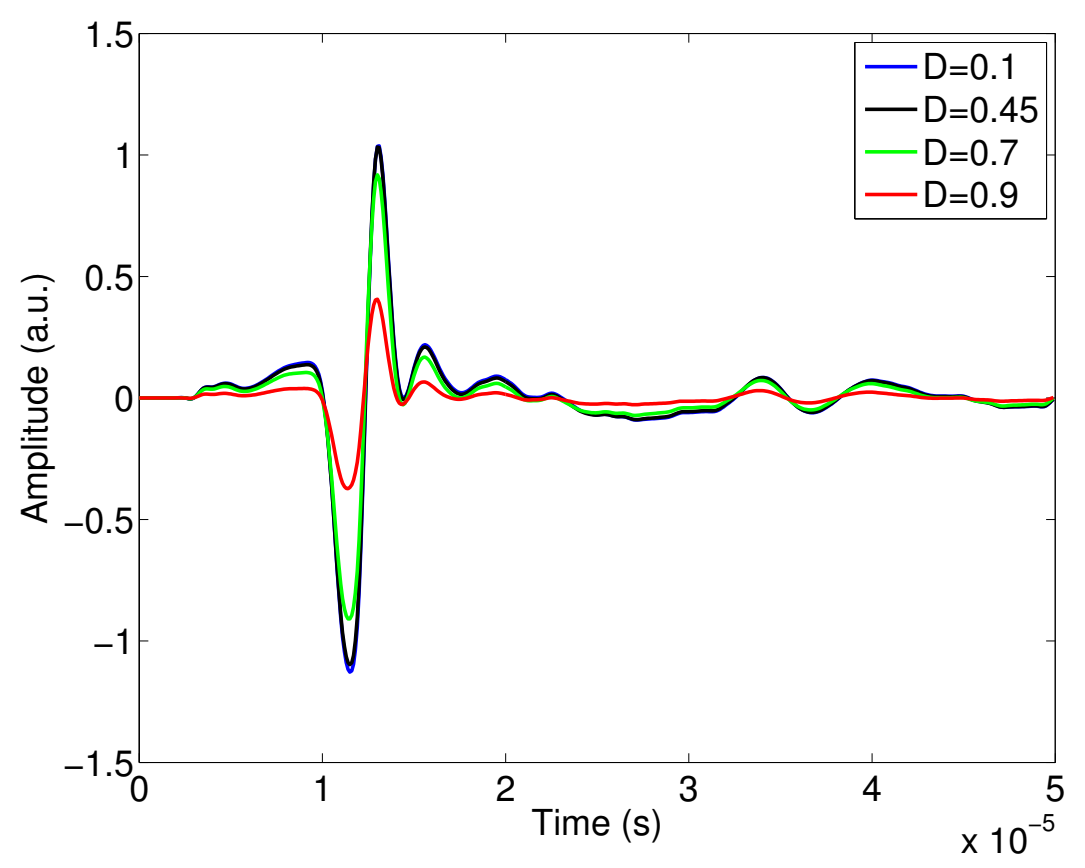


Figure 9

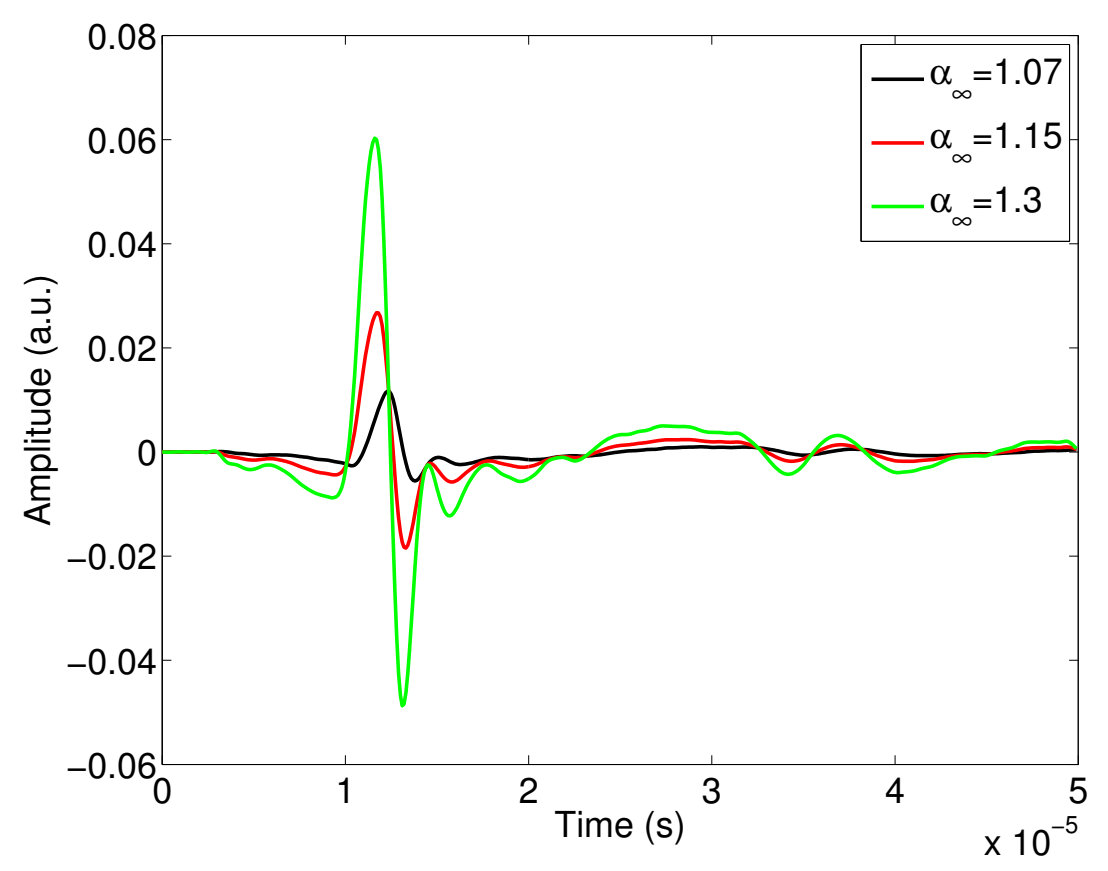


Figure 10

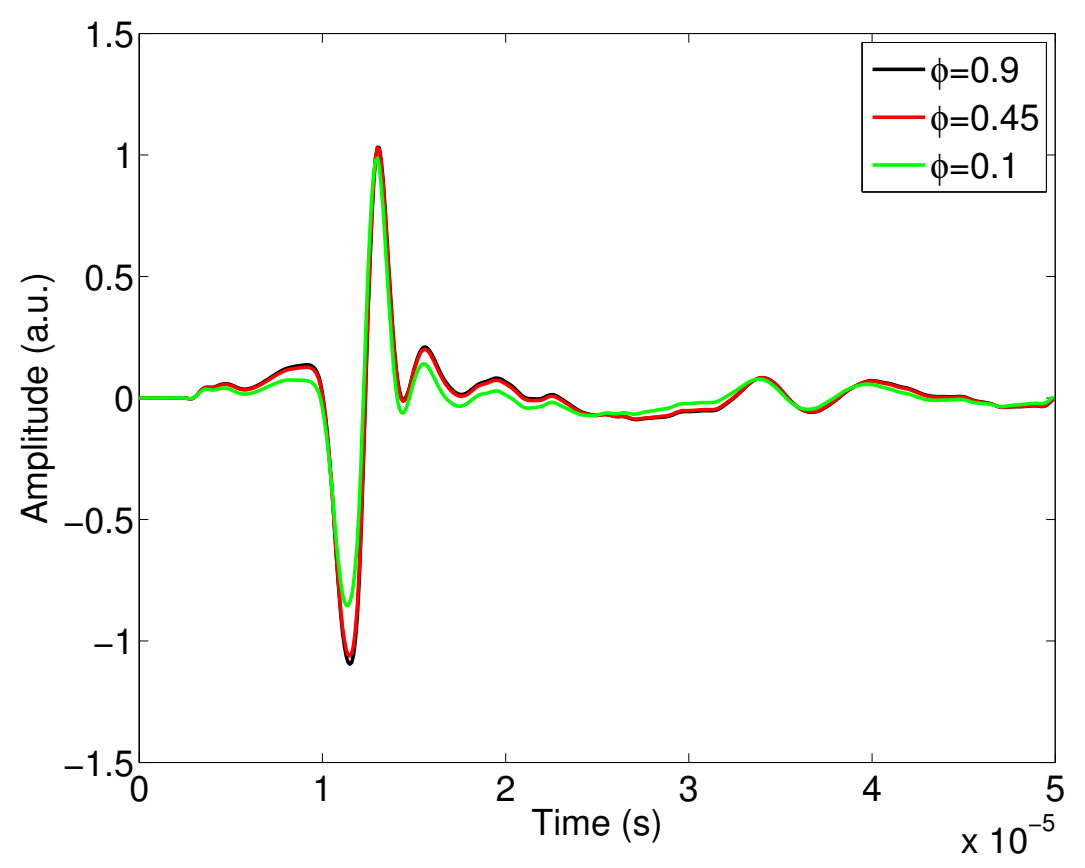


Figure 11

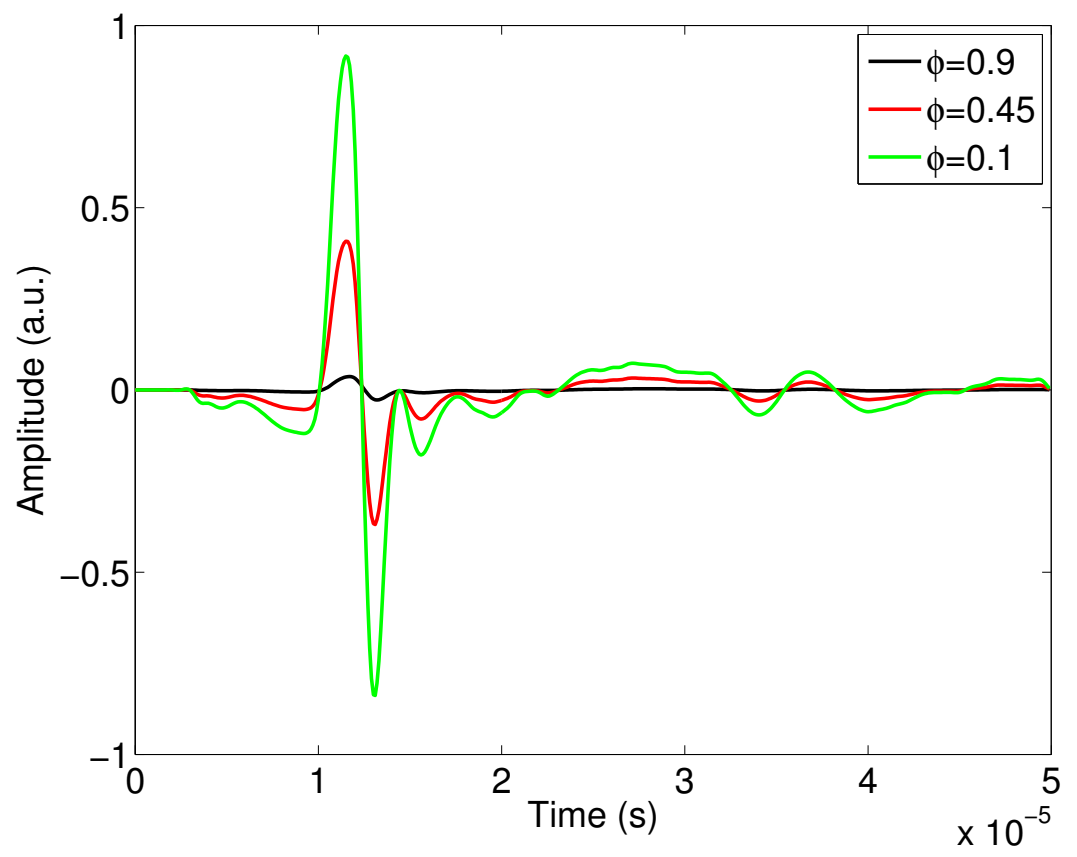

